\title{
FLUTTER DEVICE FOR RESPIRATORY DISEASES
}

\author{
B.Sarika $^{1}$, M.Shanmugapriya ${ }^{2}$, V.Suganya ${ }^{3}$, A.Velmurugan ${ }^{4}$ \\ ${ }^{1,2,3}$ Project Students, Department of Biomedical Engineering, Sri Ramakrishna Engineering College, Coimbatore, \\ India \\ ${ }^{4}$ Assistant professor (OG), Department of Biomedical Engineering, Sri Ramakrishna Engineering College, \\ Coimbatore, India \\ sarikabalasubramanian@gmail.com
}

\begin{abstract}
Respiratory diseases are now becoming as a life threatening one. Respiratory Tract is a pathway, which includes trachea, bronchi. The abnormalities or dysfunction in respiratory system may result in diseases such as bronchiectasis, cystic fibrosis, dyspnea, pulmonary fibrosis, asthma, emphysema etc. Cystic Fibrosis is an inherited disorder which is due to the abnormality in cystic fibrosis trans membrane receptor protein and it is responsible for maintaining the nature of the mucus in airway and around the pleural cavity. Bronchiectasis is an abnormality in the bronchi pathway (i.e. the airways become dilate). During this disease, hair-like projections called cilia will be enlarged and it will be unable to sweep away the mucus. Dyspnea is a condition in which the people experiencing uncomfortable changes in air pathway. In the above -mentioned diseases the common abnormality is mucus secretions stay thick. In order to overcome that Flutter device is used. Flutter is a mucus clearance device, which helps to coughed it out by performing breathing exercises. It is designed by using creo parametric 2.0 software in CAD. The $3 D$ model of the flutter is done by loading corresponding CAD file and it is loaded to the rapid prototyping machine, which prints the $3 D$ model of the flutter.
\end{abstract}

Keywords: Cystic Fibrosis, Cystic Fibrosis Transmembrane Regulator Protein, Bronchiectasis, Dyspnea, 3D Printing, Creo Parametric 2.0 etc

\section{INTRODUCTION}

Respiratory diseases affect the tubes that carry oxygen and other transport gases, which lead to oxygen starvation in surrounding tissues and the nature of the tissue, may vary. The oxygen starvation in surrounding tissue leads to dilation, inflammation in smooth tissue, air bubbles in airway, mucus secretion and emboli in respiratory tract[1]. The change in pathology of respiratory system creates pathway to lung disease like bronchitis, bronchiectasis, cystic fibrosis etc. Upper respiratory tract includes nose, throat and trachea. Bronchitis is one of the upper respiratory tract infections due to mucosal edema and the inflammation of chest wall. Generally, the upper respiratory tract infection is preceded by common cold; the other such upper respiratory disease is pneumonia common cold etc. Cystic fibrosis is one of the lower respiratory diseases, which arise due to blockage of mucus in airway and acute bronchitis comes under this condition. Awareness of mucus blockage disease in India and the availability of flutter device are very rare [1].

\subsection{BRONCHITIS}

Bronchitis is an inflammation of the bronchial tube. It leads to wheezing, chest wall tightening. While coughing mucus along the inside walls of the bronchia trap the dirt from airway with the help of cilia. Bronchitis is of two types' acute bronchitis and chronic bronchitis. Viral, bacterial or fungal organism causes acute bronchitis. The viruses that trigger bronchitis are adenovirus, influenza virus, coronavirus etc. The bacteria that cause bronchitis are bordetella pertussis, bordetella parapertussis, streptococcus pneumonia and the fungus involved are blastomyces dermatitids, Candida albicans. Normal breathing exercise and therapeutic device like flutter device helps to clean the sweep in airway [1].

\subsection{BRONCHIECTASIS}

Bronchiectasis is defined as permanent change in dilation of airway, injury in bronchial wall, blockage in bronchial lumen and by aberration in cilia functions. Cilia help to sweep the exuberance mucus in airway. Due to aberration in cilia, it cannot trap the mucus in the airway properly and leads to blockage of mucus in insides of the bronchi. The indications of bronchiectasis include coughing up blood, shortness of breath, wheezing, nail clubbing and frequent lung infection. The diagnosis depends upon the indications and confirmed using computed tomography. If bronchiectasis progresses for a long time, bacterial colonization is seen in sputum. Pathogenesis of bronchiectasis is failure of the immune system to clear infection. It is one of the chronic obstructive lung disorders that affect the immune system and give rise to various diseases [2].

\subsection{CYSTIC FIBROSIS}

Cystic Fibrosis is a critical genetic disorder which mainly affects all the system in human being especially respiratory system, digestive system, liver and pancreas. The 
indications of this disease are clubbing of nails and toes, gallstone, enlarged heart, trouble in digesting food and frequent lung infections. Cystic fibrosis patients may feel trouble to breath because of narrowing of airway and one such defect is surplus of $\mathrm{Na}-\mathrm{Cl}$ released through sweat. These irregularities are due to the abnormality in $\mathrm{Na}-\mathrm{Cl}$ transport channel. CFTR is associated with staging of sweat, digestive fluid and mucus and the main role of this regulator protein is to maintain the nature of the mucus in thin condition. The condition is diagnosed by sweat test and genetic testing. Earlier diagnostic methods such as titration procedure are used to diagnose cystic fibrosis and the drugs to treat or to cure cystic fibrosis is not available in our country. In India knowledge on cystic fibrosis is less among public. Lack of drugs and awareness among paediatricians is a big problem [3].

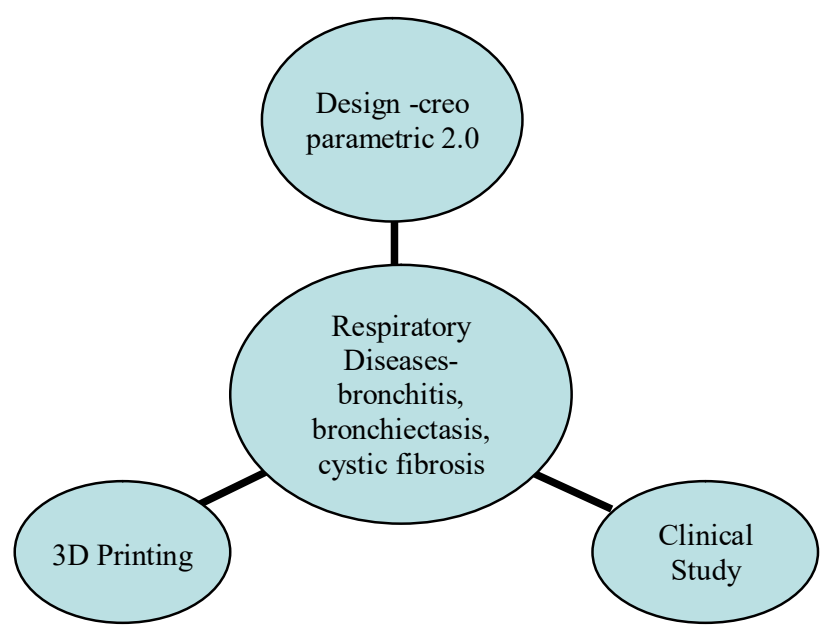

Fig 1.1 Block Diagram

\section{CREO SOFTWARE}

Creo parametric is software which serves as a best platform for product development. It mainly depends upon the features involved in creo. The definitions of the figure and the model are explained on the basis of type of features used and the order in which it is placed. Each model can refer to preceding features and build intent into an assembly [4].

\subsection{PARAMETRIC}

The parametric in creo explains about the parameters, references and the dimensions. Once the dimension is change, the corresponding geometry will be updated automatically.

\subsection{ASSEMBLY}

In creo, diverse methods are available to assemble the different parts of the figure. Assembly operation depends on which component are assembled first and the coercion that used to assemble the parts by using commands such as angle, offset etc... Each assembled part has a placement node which is located in the model pop-up menu which will tell us what constraints are used by us.

\subsection{TOOLS USED IN CREO}

A menu which is located transversely to the top of the interface which contains most of the commands in creo parametric such as extrude, revolve, sweep, swept blend, hole, round, chamfer etc .The ribbon is the one which arranges the commands by placing commands as in groups and tabs.

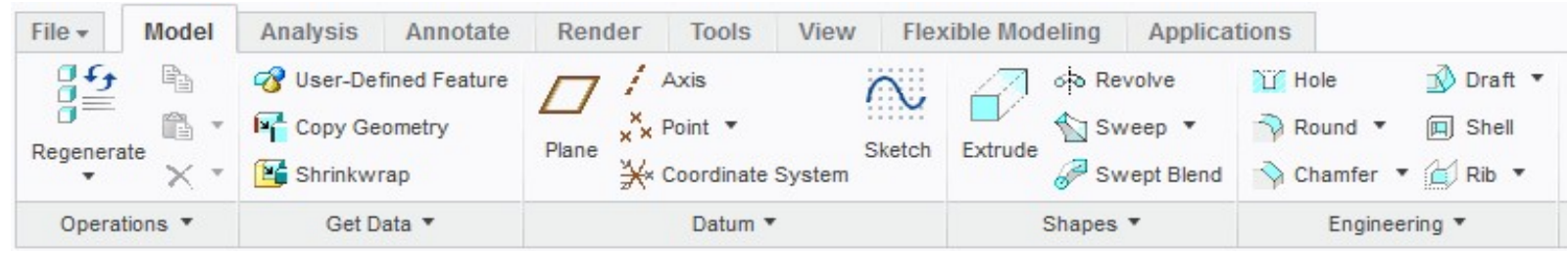

Fig 2.1 Tool palette in creo

\subsection{WORKING IN CREO}

Working directory is the start up folder for Creo parametric. It is the folder for opening and saving the files and it is selected before every session.

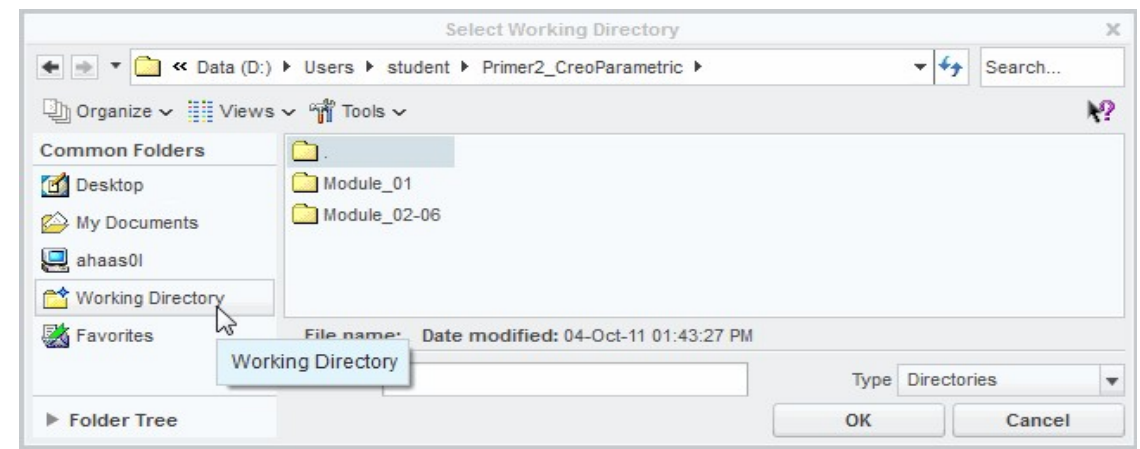

Fig 2.4 Working Directory in creo 


\subsection{MANAGING FILES IN CREO PARAMETRIC}

\subsubsection{Common File Extension}

.prt-This extension represent part files and .asm-It represents assembly files

\subsubsection{DISPLAYING STYLE OPTIONS}

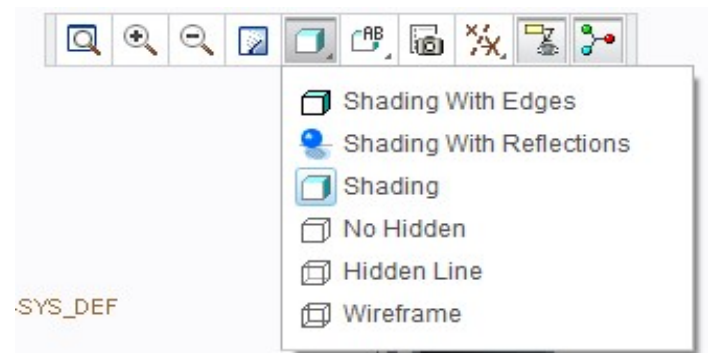

Fig 2.5.1 Style option in creo

Shading with Edges is the first displaying style in creo parametric. In this hidden lines are displayed as regular lines.

\section{FLUTTER DEVICE}

Flutter is a mucus clearance device which is used to remove the hard mucus which remains obstruction for the airway. The flutter is a part of the treatment which helps the people who have difficulty in clearing the sputum in the lungs. The flutter assembly consists of four parts namely perforated cover, steel ball, semicircular cone with mouthpiece [5].

\subsection{WORKING OF FLUTTER}

During respiration, the ball inside the flutter bounce up and down, the pressure created in flutter device produces the vibration in the human respiratory tract. The pressure along with the vibrations loosens the mucus in airway and the sputum is coughed out[5].

\subsection{DESIGN OF FLUTTER}

Design of flutter device is done by using PTC Creo parametric 2.0 released in 2012.It is developed by PTC. In this project, the design of the flutter device is done by using CAD software-Creo Parametric2.0. The design of perforated cover is designed by using revolve command and the necessity of this command is to view the object in $3 \mathrm{D}$ view. The mouthpiece with semicircular cone is also designed by using revolve, swept and swept blend command. The requisite of swept and swept blend command is to make an angle or to blend a straight line to a certain angle. The depiction of steel ball is performed by using arc command. Finally, the all parts are assembled by using assembly command [5].

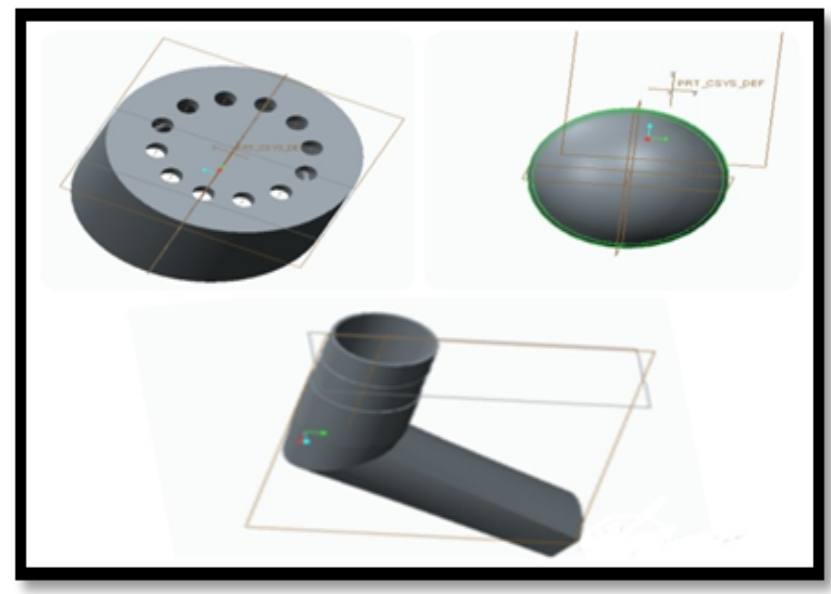

Fig 3.2.1 Parts of the flutter device

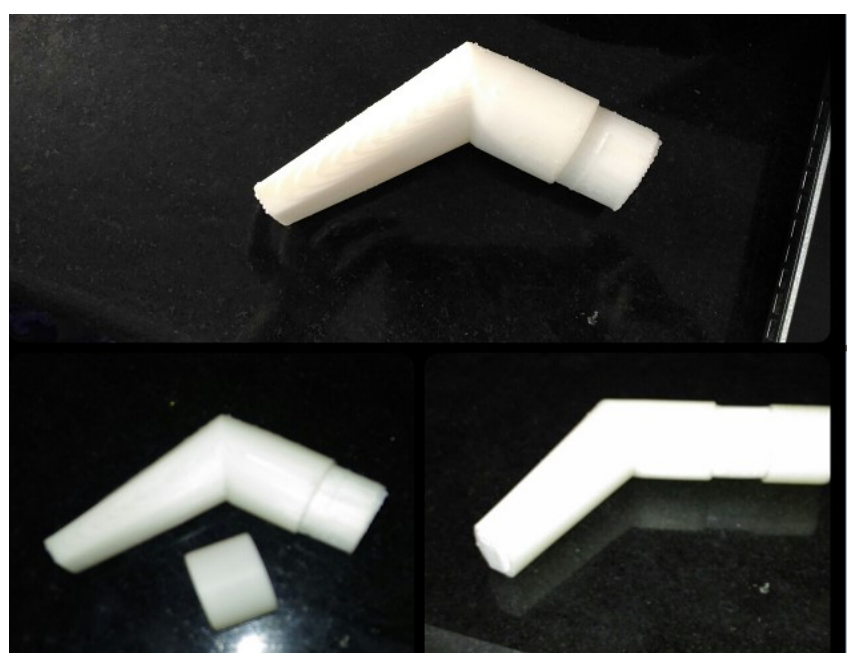

Fig 3.2.2 Prototype of flutter

\subsection{TREATMENT PROCEDURE}

Initially patient has to be relaxed and sit in a position with head tilted slightly back. Take deep inhale and hold the breath for 2 to $3 \mathrm{sec}$.Check whether the patient's stem is horizontal to the floor so that the ball can bounce. Place the flutter on the patient's mouth at a particular angle and blow out faster than normal. Blow out forcefully through the flutter and mucus is released through the cough [6].

\section{CONCLUSION}

The availability of flutter device in India is rare. This project deals with mechanical design of flutter device which is cost effective, user-friendly and portable. This will act as one of the part of treatment in respiratory disease conditions.

\section{REFERENCES}

[1] Global surveillance, prevention and control of chronic respiratory diseases: a comprehensive approach. Bousquet J, Khaltaev. World Health Organization 2007

[2] Kim JS, Muller NL, Park CS, Grenier P, Herold CJ bronchiectasis: diagnostic findings on thin- section $\mathrm{CT}$. AJR 1997; 168:751-754

[3] Cysticfibrosis.org.uk

[4] PTC Reveals Project lightning as creo, Tenlins.com 
[5] Konstan MW, Stern RC, Doershuk CF. Efficacy of the flutter device for airway mucus clearance in patients with cystic fibrosis. J Paediatrics May 1994; 124:689693.

[6] Evans AS (1967) Clinical syndromes in adults caused by respiratory infection. Med Clin North Am 51:803818 